TRANSACTIONS OF THE

AMERICAN MATHEMATICAL SOCIETY

Volume 276, Number 1, March 1983

\title{
CR-HYPERSURFACES IN A SPACE WITH A PSEUDOCONFORMAL CONNECTION
}

BY

MICHAEL J. MARKOWITZ

\begin{abstract}
In this paper we study a submanifold in a space with a pseudoconformal connection. We assume that the submanifold $M$ is so situated that it inherits the structure of a CR-hypersurface from the ambient space. $M$ then supports two natural Cartan connections, the normal pseudoconformal connection of CartanChern-Tanaka and an induced pseudoconformal connection. Analogues of the Gauss-Codazzi equations are derived and applied to determine necessary and sufficient conditions for the equivalence of these connections.
\end{abstract}

The purpose of this paper is to study an abstract CR-hypersurface immersed in a space with a pseudoconformal connection. It is shown that the connection on the ambient manifold induces a pseudoconformal connection on the submanifold in a canonical manner. One finds that this induced connection does not in general agree with the intrinsic normal connection of Cartan, Chern and Tanaka. After developing requisite background material we proceed to investigate the relationship between these connections and to derive necessary and sufficient conditions for their equality.

Of central importance in this study is a pseudoconformal analogue of the equation of Gauss in Riemannian geometry (see (5.12)). In codimension one this equation was derived independently by $\mathrm{S}$. Webster who used it to study the rigidity of hypersurfaces in a sphere [9]. In a forthcoming note written with R. Schlafly, equation (5.12) is applied to yield a negative answer to the local imbedding problem in pseudoconformal geometry. We show that the normal pseudoconformal connection on an abstract strictly pseudoconvex CR-hypersurface $M, \operatorname{dim} M \geqslant 5$, may not be realized locally by an imbedding into the unit sphere in $\mathrm{C}^{N}$ for any $N$, unless $M$ is pseudoconformally flat.

\section{Chapter 1. Pseudoconformal Geometry}

A space with a pseudoconformal connection would have been described classically as a manifold which in an immediate neighborhood of each of its points has all the properties of a real hyperquadric in complex projective space $(\$ 1)$, and upon which one is given a law permitting neighborhoods surrounding two infinitesimally close points to be "connected" in one hyperquadric. Thus one imagines that to each point of the space there is attached a tangent hyperquadric on which the point is marked and that there is given a law allowing one to view in the hyperquadric at one point,

Received by the editors September 1, 1981.

1980 Mathematics Subject Classification. Primary 32F25; Secondary 53B25, 53B15. 
the hyperquadric at another infinitesimally close point. This law defines the pseudoconformal connection and is analytically expressed by means of those infinitesimal linear fractional transformations of complex projective space which preserve a fixed hyperquadric (\$2). Accordingly the group of all such transformations plays a fundamental role in the theory of these spaces $(\$ 1)$.

Pseudoconformal connections first arose in the search for invariants of a real hypersurface in a complex manifold under the pseudogroup of local biholomorphisms. Somewhat more abstractly one may ask for the invariants of a space under maps preserving a certain $G$-structure (a CR-structure; §3). A complete system of these invariants, given by the successive covariant derivatives of the curvature forms of an intrinsic pseudoconformal connection, was first determined by E. Cartan [2] for real hypersurfaces in $\mathbf{C}^{2}$. Cartan's result was later extended to higher dimensions independently by S.-S. Chern [3] and N. Tanaka [7]. These developments are recalled in $\S 3$.

Throughout this chapter we let lower case Greek indices run from 1 to $n$, while Roman capitals run through $0,1, \ldots, n,{ }^{*}$. We also adopt standard conventions by which barred indices indicate complex conjugation and repeated indices are to be summed over their range.

1. The flat model space. Let $\zeta^{A}$ denote the homogeneous coordinates of complex projective space $\mathbf{P}^{n+1}$. In terms of these coordinates, a (nondegenerate) real hyperquadric $Q^{2 n+1}$ in $\mathbf{P}^{n+1}$ is given by the equation

$$
g_{\alpha \bar{\beta}} \zeta^{\alpha} \zeta^{\bar{\beta}}+\left(\zeta^{0} \zeta^{*}-\zeta^{0} \zeta^{\bar{*}}\right)=0
$$

where $\left(g_{\alpha \bar{\beta}}\right)$ is a nondegenerate hermitian matrix of order $n$, whose inverse will be denoted by $\left(g^{\alpha \bar{\beta}}\right)$. For the sake of convenience we will assume that $\left(g_{\alpha \bar{\beta}}\right)$ is positive definite. In this case $Q$ is diffeomorphic, in fact CR-equivalent (see $\S 3$ ), to the unit sphere $S^{2 n+1}$ in $\mathbf{C}^{n+1}$.

Let $S$ be the hermitian matrix of order $n+2$ given by

$$
S=\left(\begin{array}{ccc}
0 & 0 & -i \\
0 & g_{\alpha \bar{\beta}} & 0 \\
i & 0 & 0
\end{array}\right) .
$$

Then $Q$ is clearly the image in $\mathbf{P}^{n+1}$ of the set of vectors $Z=\left(Z_{A}\right) \in \mathbf{C}^{n+2}$ isotropic with respect to the hermitian inner product $\left\langle Z, Z^{\prime}\right\rangle=Z S^{t} \bar{Z}$ under the natural projection $\mathbf{C}^{n+2}-\{0\} \rightarrow \mathbf{P}^{n+1}$.

The group $\mathcal{L}=\mathrm{SU}(n+1,1)=\left\{A \in \mathrm{GL}(n+2, \mathrm{C}) \mid A S^{t} \bar{A}=S, \operatorname{det} A=1\right\}$ acts transitively and almost effectively on $Q$. (We shall make no attempt to distinguish

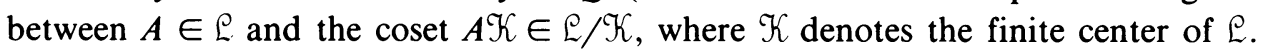
For a more careful treatment, the reader is referred to [7 or 10].) The isotropy group $\varrho_{0}$ at the origin $o=[(1,0, \ldots, 0)] \in Q$ is the semidirect product $\mathrm{CU}(n) \propto N^{+}$of the conformal unitary group with the Heisenberg group of dimension $2 n+1$. We may now identify the homogeneous space $Q$ with the space of cosets $\varrho / L_{0}$. 
The Lie algebra $\mathfrak{l}=s u(n+1,1)$ of $\mathcal{L}$ has the structure of a simple graded Lie algebra [7]. To see this we set

$$
\begin{aligned}
& \mathfrak{g}_{-2}=\left\{\left(\begin{array}{ccc}
0 & 0 & r \\
0 & 0 & 0 \\
0 & 0 & 0
\end{array}\right)\right\}, \quad g_{-1}=\left\{\left(\begin{array}{ccc}
0 & u^{\beta} & 0 \\
0 & 0 & i g_{\alpha \bar{\beta}} u^{\bar{\beta}} \\
0 & 0 & 0
\end{array}\right)\right\}, \\
& \mathfrak{g}_{0}=\left\{\left(\begin{array}{ccc}
z & 0 & 0 \\
0 & A_{\alpha}^{\beta} & 0 \\
0 & 0 & -\bar{z}
\end{array}\right) \mid \begin{array}{c}
g_{\beta \bar{\gamma}} A_{\alpha}^{\beta}+\bar{A}_{\gamma}^{\beta} g_{\alpha \bar{\beta}}=0 \\
z-\bar{z}+A_{\alpha}^{\alpha}=0
\end{array}\right\}, \\
& g_{1}=\left\{\left(\begin{array}{ccc}
0 & 0 & 0 \\
-i g_{\alpha \bar{\beta}} v^{\bar{\beta}} & 0 & 0 \\
0 & v^{\beta} & 0
\end{array}\right)\right\}, \quad g_{2}=\left\{\left(\begin{array}{ccc}
0 & 0 & 0 \\
0 & 0 & 0 \\
s & 0 & 0
\end{array}\right)\right\},
\end{aligned}
$$

where $r, s \in \mathbf{R}$ and $u^{\alpha}, v^{\alpha}, A_{\alpha}^{\beta}, z \in \mathbf{C}$. Then $l=\mathfrak{g}_{-2}+\mathfrak{g}_{-1}+\mathfrak{g}_{0}+\mathfrak{g}_{1}+\mathfrak{g}_{2}$ and, if we understand that $\mathfrak{g}_{k}=\{0\}$ for $k<-2$ or $k>2,\left[\mathfrak{g}_{i}, \mathfrak{g}_{j}\right] \subseteq \mathfrak{g}_{i+j}$. The Lie algebra of $\varrho_{0}$ is the graded subalgebra $\mathfrak{l}_{0}=\mathfrak{g}_{0}+\mathfrak{g}_{1}+\mathfrak{g}_{2}$.

It is a standard fact in the theory of homogeneous spaces $[4,6]$ that the tangent space $m$ at $o \in Q$ may be naturally identified with $\mathfrak{l} / \mathfrak{l}_{0}$. Under this identification the adjoint representation of $\mathfrak{L}_{0}$ on $\mathfrak{l} / \mathfrak{l}_{0}$ coincides with the linear isotropy representation $\rho: \mathcal{L}_{0} \rightarrow \mathrm{GL}(\mathrm{m})$. Note that the subgroup $\mathcal{S}_{2} \subseteq \mathcal{L}_{0}$ generated by $\mathfrak{g}_{2}$ is in the kernel of $\rho$. In fact, identifying $\mathfrak{l} / \mathfrak{l}_{0}$ with $\mathfrak{g}_{-2}+\mathfrak{g}_{-1}$, one may show that the linear isotropy group $\mathfrak{L}_{0}^{\prime}=\rho\left(\mathfrak{I}_{0}\right) \subseteq \mathrm{GL}(\mathfrak{m})$ is the semidirect product $\mathrm{CU}(n) \propto \mathrm{C}^{n}[1,7,10]$.

A projective frame for $Q$ is an ordered set $\left\{Z_{A}\right\}$ of $n+2$ vectors in $\mathbf{C}^{n+2}$ such that $\left(\left\langle Z_{A}, Z_{B}\right\rangle\right)=S$. A normalized projective frame or $Q$-frame [3] is a projective frame $\left\{Z_{A}\right\}$ which satisfies $Z_{0} \wedge \cdots \wedge Z_{*}=1$. Note that $Z_{0}$ and $Z_{*}$ may be regarded as points on $Q$.

Let $Y$ denote the space of all $Q$-frames and define a projection $\pi: Y \rightarrow Q$ by setting $\pi\left(\left\{Z_{A}\right\}\right)=Z_{0}$. It is not hard to see that $\mathcal{L}$ acts simply transitively on $Y$. Thus we may identify $\mathcal{L}$ and $Y$ once a reference $Q$-frame has been specified. In fact, $Y$ is a principal $\mathcal{L}_{0}$-bundle over $Q$ and choosing a frame over $o \in Q, \pi$ may be identified with the natural projection $\mathcal{L} \rightarrow \mathcal{L} / \mathcal{L}_{0}=Q$.

REMARK. We shall see in $\S 2$ that the quotient $Y / \mathcal{G}_{2}$ may be viewed as an $\mathcal{E}_{0}^{\prime}$-subbundle of the bundle of linear frames on $Q$. Thus $Q$ carries a natural $\mathcal{L}_{0}^{\prime}$-structure which is essentially just the CR-structure it inherits as a real hypersurface in $\mathbf{P}^{n+1}$ (§3). In fact, represented as $\mathscr{L} / \mathscr{L}_{0}, Q$ is the semisimple flat homogeneous model space for pseudoconformal geometry.

2. Pseudoconformal connections. We are now in a position to formalize one of the notions discussed in the introduction.

(2.1) Definition. A pseudoconformal connection on a manifold $M$ of dimension $2 n+1$ is a Cartan connection of type $\mathcal{L} / \mathcal{L}_{0}$ on $M$. Such a connection consists of a principal $\varrho_{0}$-bundle $P$ over $M$, together with an l-valued l-form $\omega$ on $P$ satisfying

(i) $R_{a}^{*} \omega=\operatorname{ad}\left(a^{-1}\right) \omega$ for $a \in \mathcal{L}_{0}$,

(ii) $\omega\left(A^{*}\right)=A$ for $A \in \mathfrak{l}_{0}$,

(iii) $\omega(X) \neq 0$ for $X \neq 0, X$ tangent to $P$. 
Here $R_{a}$ denotes the right action of $a \in \mathscr{L}_{0}$ on $P, \operatorname{ad}\left(a^{-1}\right)$ the adjoint representation of $a^{-1}$ on $\mathfrak{l}$, and $A^{*}$ the fundamental vector field on $P$ corresponding to $A \in \mathfrak{l}_{0}$.

The curvature $\Omega$ of a pseudoconformal connection $\omega$ is the $\mathfrak{l}$-valued 2 -form on $P$ defined via the structure equation

$$
d \omega=\omega \wedge \omega+\Omega .
$$

We say that the connection $\omega$ is flat if $\Omega=0$.

EXAMPLE. The left-invariant Maurer-Cartan form on $\mathcal{E}$ is easily seen to be a flat pseudoconformal connection in the bundle $Y \rightarrow Q$. Conversely, as a standard application of the Frobenius theorem shows, a manifold with a flat pseudoconformal connection is locally equivalent to $Q$ with this standard connection.

Let $\omega_{i}$ (resp. $\Omega_{i}$ ) denote the graded components of $\omega$ (resp. $\Omega$ ) with respect to the decomposition (1.1). $\Omega_{-2}+\Omega_{-1}$ is often called the torsion of $\omega$. In the sequel we shall only be concerned with torsionfree connections, i.e. connections for which $\Omega_{-2}+\Omega_{-1}=0$. For such a connection, the $g_{-2^{-}}$and $g_{-1}$-components of $d \omega$ given by the structure equation (2.2) may be written more explicitly (in the usual basis for l) as

$$
\begin{aligned}
& d \omega_{0}^{*}=i g_{\alpha \bar{\beta}} \omega_{0}^{\alpha} \wedge \bar{\omega}_{0}^{\beta}+\omega_{0}^{*} \wedge\left(\omega_{*}^{*}-\omega_{0}^{0}\right), \\
& d \omega_{0}^{\alpha}=\omega_{0}^{\beta} \wedge\left(\omega_{\beta}^{\alpha}-\delta_{\beta}^{\alpha} \omega_{0}^{0}\right)+\omega_{0}^{*} \wedge \omega_{*}^{\alpha} .
\end{aligned}
$$

From the properties (2.1)(i),(ii) one derives the standard fact that the curvature form $\Omega$ is quadratic in the basic forms $\omega_{0}^{*}, \omega_{0}^{\alpha}, \bar{\omega}_{0}^{\beta}$ (see [5, p. 129]). The trace of any such form

$$
\theta \equiv a_{\alpha \bar{\beta}} \omega_{0}^{\alpha} \wedge \bar{\omega}_{0}^{\beta} \bmod \left(\omega_{0}^{*}, \omega_{0}^{\rho} \wedge \omega_{0}^{\sigma}, \omega_{0}^{\rho} \wedge \bar{\omega}_{0}^{\sigma}\right)
$$

is defined by $\operatorname{Tr} \theta=g^{\alpha \bar{\beta}} a_{\alpha \beta}$ [3, p. 263]. A torsionfree pseudoconformal connection $\omega$ on $M$ is said to be normal if $\operatorname{Tr} \Omega_{\alpha}^{\beta}=\operatorname{Tr} \Omega_{\alpha}^{0}=\operatorname{Tr} \Omega_{0}^{0}=\operatorname{Tr} \Omega_{*}^{0}=0$. Using the Bianchi identities for $\omega, \mathrm{S}$. Webster has in fact shown that under these conditions $\Omega_{0}^{0}=\Omega_{*}^{*}=0$ ([8] and the appendix of [3]). (A somewhat different, though equivalent, basis free formulation of this normality condition may be found in [7].)

We shall now indicate how a pseudoconformal connection gives rise to a geometric structure on the base space $M$. More details may be gleaned from [7 or 10].

First note that $\omega_{-}=\omega_{-2}+\omega_{-1}$ is a vector valued 1 -form on $P$ satisfying $R_{a}^{*} \omega_{-}=\rho\left(a^{-1}\right) \omega_{-}$for $a \in \mathcal{L}_{0}$, where $\rho$ denotes the linear isotropy representation of $\mathcal{L}_{0}$ on $\mathfrak{g}_{-2}+\mathfrak{g}_{-1}$ as in $\S 1$. The quotient $P / \mathcal{G}_{2}$ of $P$ by the kernel of $\rho$ is a principal $\mathcal{L}_{0}^{\prime}$-bundle over $M$. It is not hard to see that $\omega_{-}$"projects" onto a unique $\mathfrak{g}_{-2}+\mathfrak{g}_{-1}$-valued 1-form, also denoted by $\omega_{-}$, on $P / \mathcal{G}_{2}$ which satisfies (2.1)(i) for $a \in \mathcal{L}_{0}^{\prime}$. Since $\mathcal{L}_{0}^{\prime}=\mathrm{CU}(n) \propto \mathbf{C}^{n} \subseteq \mathrm{GL}\left(\mathfrak{g}_{-2}+\mathfrak{g}_{-1}\right)$, the basic form $\omega_{-}$allows us to identify $P / \mathcal{S}_{2}$ with a subbundle of the bundle of linear frames on $M$. One obtains an $\mathcal{L}_{0}^{\prime}$-structure on $M$ in this manner.

A somewhat different, but equivalent, approach goes as follows. Let $P^{\mathcal{L}}=P \times_{\mathfrak{E}_{0}} \mathcal{L}$

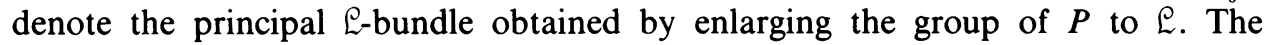
quotient $B=P^{\mathfrak{E}} / \mathcal{L}_{0}$ is a fiber bundle over $M$ with structure group $\mathcal{L}$ and fiber 
$\mathcal{L} / \mathscr{L}_{0}=Q$. (The structure group of $B$ can of course be reduced to $\mathscr{L}_{0}$.) Note that $B \rightarrow M$ has a natural section $\sigma$ given as the image of $P$ under the projection $P^{\mathfrak{L}} \rightarrow B$. It is well known that the basic form (or solder form) $\omega_{-}$may be viewed as a bundle isomorphism $\tau: T M \rightarrow \sigma^{*} T_{F} B$, where $T_{F} B$ denotes the bundle of vectors tangent to the fibers of $B$ [5]. The natural $\mathcal{L}_{0}^{\prime}$-structure on $Q$ may now be transferred to $M$ via $\tau$.

Classically one identifies $x \in M$ with $\sigma(x) \in B_{x}$ and speaks of the fiber $B_{x}=Q$ as being tangent to $M$ at $x$. This is the "tangent hyperquadric" referred to in the introduction. Since each fiber $P_{x}$ of $P$ may be identified with the collection of $Q$-frames at $\sigma(x)$, we refer to $P$ as the bundle of $Q$-frames on $M$.

3. CR-hypersurfaces. In this section we shall review some results concerning the geometric structure inherited by a "generic" real hypersurface in a complex manifold. We recommend that the reader consult the survey article [1] for additional information.

The structure of an abstract real hypersurface [3] (an almost CR-hypersurface or pseudocomplex structure [7]) on a manifold $M$ of dimension $2 n+1$ is given locally by $2 n+1$ linearly independent complex valued 1 -forms $\theta=\bar{\theta}, \theta^{\alpha}, \theta^{\bar{\beta}}=\overline{\theta^{\beta}}$, determined up to the transformation

$$
\begin{array}{ll}
\theta^{\prime}=t \theta, & t \in \mathbf{R}, v^{\alpha} \in \mathbf{C}, \\
\theta^{\alpha^{\prime}}=u_{\beta}^{\alpha} \theta^{\beta}+v^{\alpha} \theta, & \\
\theta^{\beta^{\prime}}=u_{\bar{\gamma}}^{\bar{\beta}} \theta^{\bar{\gamma}}+v^{\bar{\beta}} \theta, \quad u_{\beta}^{\alpha} \in \mathrm{GL}(n, \mathbf{C}), u_{\bar{\beta}}^{\bar{\alpha}}=\overline{u_{\beta}^{\alpha}} .
\end{array}
$$

Thus we have a $\mathcal{G}$-structure on $M$, where $\mathcal{G}$ is the group of nonsingular matrices of the form

$$
\left(\begin{array}{ccc}
t & 0 & 0 \\
v^{\alpha} & u_{\beta}^{\alpha} & 0 \\
v^{\bar{\alpha}} & 0 & u_{\bar{\beta}}^{\bar{\alpha}}
\end{array}\right)
$$

A pseudocomplex structure is said to be integrable if the ideal of complex valued forms generated by $\theta, \theta^{\alpha}$ is closed. In this case, since $\theta$ is real, we may write

$$
\begin{aligned}
d \theta & \equiv i g_{\alpha \beta^{-} \theta^{\alpha} \wedge \theta^{\bar{\beta}}} \quad \bmod \theta \\
d \theta^{\alpha} & \equiv \theta^{\beta} \wedge \omega_{\beta}^{\alpha}
\end{aligned}
$$

where $g_{\alpha \bar{\beta}}=\overline{g_{\bar{\beta} \alpha}}=g_{\beta \bar{\alpha}}$. An integrable pseudocomplex structure is nondegenerate if the hermitian matrix $\left(g_{\alpha \bar{\beta}}\right)$ is nonsingular. One sees that this notion is well defined by checking that, under the change (3.1), the Levi form $g_{\alpha \beta}$ undergoes the transformation

$$
g_{\alpha \overline{\beta^{\prime}}}=t u_{\alpha}^{\gamma} g_{\gamma \bar{\sigma}} u_{\bar{\beta}}^{\bar{\sigma}} .
$$

A nondegenerate, integrable pseudocomplex structure will be called a $C R$-structure on $M$, although this terminology is usually applied to a more general situation. We shall call a manifold with such a CR-structure a $C R$-hypersurface.

Note that at each point $x \in M$ the real codimension-one subspace $H_{x} M$ of $T_{x} M$, consisting of tangent vectors annihilated by $\theta$, is well defined by (3.1) and has a 
natural complex structure. We call $H_{x} M$ the complex tangent space at $x$. The union of the $H_{x} M$ as $x$ ranges over $M$ is the complex tangent bundle $H M$. The complex structures on the fibers of $H M$ fit together to give a bundle endomorphism $J$ : $H M \rightarrow H M$, such that $J^{2}=-I$. The integrability condition may be expressed in terms of $J$ as

$$
[X, Y]-[J X, J Y]-J([J X, Y]+[X, J Y])=0
$$

for all sections $X, Y$ of $H$.

If the Levi form of $M$ is positive (or negative) definite, $M$ is said to be strongly pseudoconvex. In this case it is apparent from (3.4) that the structure group of $M$ may be reduced to $\mathcal{L}_{0}^{\prime}=\mathrm{CU}(n) \propto \mathbf{C}^{n} \subseteq \mathcal{G}$ by requiring that $g_{\alpha \bar{\beta}}=\delta_{\beta}^{\alpha}$. Conversely, if $M$ is a manifold with an $\mathcal{L}_{0}^{\prime}$-structure, then $M$ has an underlying pseudocomplex structure obtained by enlarging the structure group to $\mathcal{G}$.

Recalling the discussion of the previous section and comparing (2.3) with (3.2) and (3.3) yields the following

(3.6) Proposition. A torsionfree pseudoconformal connection on a manifold $M$ naturally induces a (nondegenerate, integrable) CR-structure on $M$.

The CR-structure on $Q$ induced in this way by the Maurer-Cartan form $\pi$ on $\mathcal{L}=Y$ coincides with the structure it inherits as a real hypersurface in $\mathbf{P}^{n+1}$. It is well known that the group of automorphisms of this structure is $\mathcal{L}$ (actually $\mathcal{L} / \mathcal{K}$ ). In fact, $\mathcal{E}$ is also the full group of bundle automorphisms of $Y \rightarrow Q$ preserving the connection $\pi$. It is in this sense that $(Y, \pi)$ solves the "equivalence problem" for this G-structure on $Q$.

The fundamental theorem in pseudoconformal geometry, being the converse of (3.6), is the following generalization of this situation to general CR-hypersurfaces.

(3.7) Theorem (CARTAN [2], Chern [3], TANAKA [7]). Let $M$ be a (nondegenerate, integrable) CR-hypersurface. There exists a unique, intrinsically defined, normal pseudoconformal connection $(P, \omega)$ on $M$. Furthermore, a diffeomorphism $f: M \rightarrow M$ is a CR-automorphism if and only if there is a bundle automorphism $\tilde{f}$ of $P, \tilde{f}$ covering $f$, which preserves the connection in the sense that $\tilde{f}^{*} \omega=\omega$.

One consequence of this result is the fact that the group of CR-automorphisms of a nondegenerate, integrable CR-hypersurface is a Lie transformation group of dimension at most $n^{2}+4 n+3=\operatorname{dim} \mathcal{L}$, if $\operatorname{dim} M=2 n+1$.

\section{Chapter 2. CR-SUbmanifolds}

Having defined the notion of a space with a pseudoconformal connection in the previous chapter, we now turn to the study of submanifolds in such a space. In order to obtain a meaningful theory, attention must be restricted to those submanifolds whose tangent spaces lie in a fixed relationship with the CR-structure of the ambient space. The submanifolds which appear to us to lead most naturally to interesting results are the subobjects in our category. Thus we require our submanifolds to be "maximally complex" and consequently to carry an induced CR-structure. We should, however, point out that the opposite extreme, comprised of totally real or 
"anti-invariant" submanifolds, has received much more attention in the literature (one notable exception is [9]).

We begin with the following

Definition. Let $V$ be a $2 N+1$-dimensional manifold with a (torsionfree) pseudoconformal connection. A CR-hypersurface $M$, of dimension $2 n+1(n<N)$, is said to be pseudoconformally immersed in $V$ if there is given an immersion $\iota$ : $M \rightarrow V$ such that

$$
\begin{array}{ll}
\text { (i) } & \iota_{*} T M+H V=T V, \\
\text { (ii) } & \iota_{*} H M \subseteq H V, \\
\text { (iii) } & \iota_{*} J_{M}=J_{V} \iota_{*} .
\end{array}
$$

We set $N=n+p$ and call $p$ the (complex) codimension of $M$ in $V$.

Throughout the remainder of this paper $M$ will denote a CR-hypersurface of dimension $2 n+1$ pseudoconformally immersed in a $2 N+1$-dimensional space $V$ with a torsionfree pseudoconformal connection as above. Recall that we are tacitly assuming that $M$, as well as $V$, is strongly pseudoconvex. The general case may be treated similarly if one stipulates that the Levi form of $V$ restricts to a nondegenerate form on $H M$.

We shall see in $\S 6$ that the connection on $V$ induces a pseudoconformal connection on $M$ which in general does not agree with the intrinsic normal connection given by (3.7). In this chapter we derive pseudoconformal analogues of the classical equations of Gauss and Codazzi in order to study the relationship between these connections and, in particular, to find necessary and sufficient conditions for their equality. Our treatment follows the discussion of submanifolds in a space with a (normal) conformal connection found in [11]. (See [9] for a similar development for the case $p=1$.)

We adopt the following conventions regarding the ranges of various indices which will appear:

$$
\begin{aligned}
A, B, C, \ldots & =0,1, \ldots, n+p,^{*}, \\
J, K, L, \ldots & =0,1, \ldots, n,^{*}, \\
i, j, k, \ldots & =1, \ldots, n, \\
P, Q, R, \ldots & =n+1, \ldots, n+p, \\
\alpha, \beta, \gamma, \ldots & =1, \ldots, n+p, \\
\mu, \nu, \xi, \ldots & =1, \ldots, n+p,^{*}, \\
\rho, \sigma, \tau, \ldots & =1, \ldots, n,{ }^{*} .
\end{aligned}
$$

The Levi form will also be used to raise and lower indices as follows:

$$
\begin{gathered}
g^{i j} T_{\bar{j}}^{P m}=g^{i \bar{j}} \bar{T}_{j}^{\bar{P} \bar{m}}=T^{i P m}, \quad g_{i \bar{j}} W_{k}^{i t}=W_{k \bar{j}}^{l}, \\
g^{A \bar{B}} X_{i A \bar{B}}^{j}=X_{i A}^{j A}, \quad \text { etc. }
\end{gathered}
$$

4. Adapted frames and the second fundamental form. A $Q$-frame $\left(Z_{A}\right)=$ $\left(Z_{0}, Z_{i}, Z_{P}, Z_{*}\right)$ for $V$, at a point of $M$, is said to be adapted to $M$ if $\left(Z_{0}, Z_{i}, Z_{*}\right)$ is 
a $Q$-frame for $M$. In other words, $Z_{0}$ lies on $M$ and the $Z_{i}$ span the complex tangent space $H_{Z_{0}} M$. Moreover, since the hyperquadric $Q_{0}$ tangent to $M$ at $Z_{0}$ is the intersection of the hyperquadric tangent to $V$ with a linear subspace of the ambient projective space (see 4.4), it makes sense to require that $Z_{*}$ lie on $Q_{0}$. Finally, the $Z_{P}$ are chosen normal to $M$.

We normalize our adapted frames by requiring that the hermitian matrix $g_{A \bar{B}}=$ $\left\langle Z_{A}, Z_{B}\right\rangle$ be of the form

$$
\left(\begin{array}{cccc}
0 & 0 & 0 & -i \\
0 & g_{i j} & 0 & 0 \\
0 & 0 & I_{P} & 0 \\
i & 0 & 0 & 0
\end{array}\right)
$$

Here $g_{i j}-$ is a (positive definite) hermitian matrix of order $n$ and $I_{P}$ denotes the identity matrix of order $p$. It is easy to check that the (normalized) adapted frames are determined up to a transformation of the form

$$
U=\left(\begin{array}{cccc}
t & 0 & 0 & 0 \\
t_{i} & t_{i}^{j} & 0 & 0 \\
0 & 0 & t_{P}^{Q} & 0 \\
\tau & \tau^{j} & 0 & \bar{t}^{-1}
\end{array}\right)
$$

where $U$ acts on $\left(Z_{A}\right)$ on the left and the following identities are satisfied:

$$
\begin{gathered}
i \bar{t}^{-1} t_{t}=-\tau^{j} g_{j \bar{k}} \bar{t}_{l}^{k}, \quad \bar{t}^{-1} \operatorname{det}\left(t_{i}^{j}\right) \operatorname{det}\left(t_{P}^{Q}\right)=1, \quad t_{i}^{j} g_{j k} \bar{t}_{t}^{k}=g_{i \bar{t}}, \\
g_{j k} \tau^{j} \tau^{\bar{k}}+i\left(\bar{t}^{-1} \bar{\tau}-\tau t^{-1}\right)=0, \quad t_{P}^{Q} \bar{t}_{R}^{Q}=\delta_{P}^{R} .
\end{gathered}
$$

If $d Z_{A}=\pi_{A}^{B} Z_{B}$ defines the normal pseudoconformal connection on $V$ with respect to the adapted frame field $Z_{A}$, then

$$
\pi_{0}^{P}=0
$$

along $M$, since $Z_{0}$ is restricted to lie on $M$. Furthermore, the connection $\pi=\left(\pi_{A}^{B}\right)$ satisfies

$$
\begin{array}{ll}
\pi_{A}^{C} g_{C \bar{B}}+g_{A \bar{C}} \bar{\pi}_{B}^{C}=0, & \pi_{A}^{A}=0, \\
d \pi_{A}^{B}=\pi_{A}^{C} \wedge \pi_{C}^{B}+\Pi_{A}^{B}, & \Pi_{A}^{B}=C_{A \mu \nu}^{B} \pi_{0}^{\mu} \wedge \bar{\pi}_{0}^{\nu} .
\end{array}
$$

The condition of normality on $\pi$ to be satisfied by the curvature components $C_{A \mu \bar{\nu}}^{B}$ is that

$$
g^{\mu \bar{\nu}} C_{A \mu \bar{\nu}}^{B}=0 .
$$

Also note that $C_{A \mu \bar{\nu}}^{B}=0$ if either $A=0$, or $B={ }^{*}$. 
Taking into account the special form of (4.1), we may write out the first equation of (4.5) somewhat more explicitly as

$$
\begin{aligned}
& \pi_{0}^{*}-\bar{\pi}_{0}^{*}=0, \quad \pi_{i}^{*}=i g_{i j} \bar{\pi}_{0}^{j}, \quad \pi_{P}^{*}=i \bar{\pi}_{0}^{P}, \\
& \pi_{0}^{0}+\bar{\pi}_{*}^{*}=0, \quad \pi_{i}^{j} g_{j \bar{k}}=-g_{i j} \bar{\pi}_{k}^{j}, \quad \pi_{P}^{Q}=-\bar{\pi}_{Q}^{P}, \\
& \pi_{*}^{0}-\bar{\pi}_{*}^{0}=0, \quad \pi_{i}^{0}=-i g_{i \bar{k}} \bar{\pi}_{*}^{k}, \quad \pi_{P}^{0}=-i \bar{\pi}_{*}^{P} . \\
& \pi_{i}^{P}=-g_{i k} \bar{\pi}_{P}^{k},
\end{aligned}
$$

The above equations will often be used implicitly in the calculations which follow.

Now let $\omega^{i}, \omega^{*}=\bar{\omega}^{*}$ be the complex 1-forms of an admissible coframe for the CR-structure on $M$, so that $d \omega^{*}=i g_{i j} \omega^{i} \wedge \bar{\omega}^{j}+\omega^{*} \wedge \phi$ for some real l-form $\phi$. Let $\omega=\left(\omega_{J}^{K}\right)$ denote the normal pseudoconformal connection on $M$, pulled down from $P$ via this coframe field, so that $\omega_{0}^{*}=\omega^{*}$ and $\omega_{0}^{i}=\omega^{i}$. $\omega$ satisfies conditions similar to those in (4.5) and (4.6).

Under a change of adapted frames as in (4.2), $\pi$ undergoes the transformation $\tilde{\pi} U=U \pi+d U$. In particular, we have

$$
\begin{gathered}
\bar{t}^{-1} \tilde{\pi}_{0}^{*}=t \pi_{0}^{*}, \quad \tilde{\pi}_{0}^{j} t_{j}^{i}+\tau \tilde{\pi}_{0}^{*}=t \pi_{0}^{i}, \\
t \tilde{\pi}_{0}^{0}+\tilde{\pi}_{0}^{j} t_{j}+\tau \tilde{\pi}_{0}^{*}=t \pi_{0}^{0}+d t
\end{gathered}
$$

on $M$. It is apparent from (4.7) that we may choose $U$ in such a way that

$$
\pi_{0}^{*}=\omega^{*} \text { and } \pi_{0}^{j}=\omega^{j} \text {. }
$$

Comparing $d \pi_{0}^{*}=i g_{i j} \pi_{0}^{i} \wedge \bar{\pi}_{0}^{j}+\pi_{0}^{*} \wedge\left(\pi_{*}^{*}-\pi_{0}^{0}\right)$ with the corresponding equation for $d \omega_{0}^{*}$ and using (4.9), one sees that

$$
\pi_{0}^{0}-\pi_{*}^{*}=\pi_{0}^{0}+\bar{\pi}_{0}^{0}=\omega_{0}^{0}+\bar{\omega}_{0}^{0}+s \omega^{*} .
$$

Taking $t=1, t_{i}^{j}=\delta_{i}^{j}, t_{P}^{Q}=\delta_{P}^{Q}, \tau=s / 2$ in (4.2) and using (4.8), we may further assume that

$$
\pi_{0}^{0}+\bar{\pi}_{0}^{0}=\omega_{0}^{0}+\bar{\omega}_{0}^{0} .
$$

Note that (4.9) and (4.10) provide expressions for the $g_{-2}, g_{-1}$, and one of the $\mathfrak{g}_{0}$-components of the connection $\pi$ in terms of the intrinsic connection $\omega$. Our immediate aim is to find similar expressions for the remaining components of this connection.

The exterior derivative of (4.4) may be written as $0=d \pi_{0}^{P}=\omega^{i} \wedge \pi_{i}^{P}+\omega^{*} \wedge \pi_{*}^{P}$. Cartan's lemma asserts that

$$
\pi_{i}^{P}=H_{i j}^{P} \omega^{j}+H_{i}^{P} \omega^{*}, \quad \pi_{*}^{P}=H_{j}^{P} \omega^{j}+H^{P} \omega^{*}
$$

with $H_{i j}^{P}=H_{j i}^{P}$. The forms (4.11) comprise the second fundamental form of the immersion $\iota: M \rightarrow V$. We say that $M$ is auto-parallel in $V$ if $\pi_{i}^{P}=\pi_{*}^{P}=0$. (We prefer this terminology to the "totalement ombilique" applied to the analogous condition in [11].) 
The exterior derivative of the first equation of (4.9) has already been used. The exterior derivative of the second equation, however, yields

$$
0=\omega^{i} \wedge\left\{\left(\omega_{i}^{j}-\delta_{i}^{j} \omega_{0}^{0}\right)-\left(\pi_{i}^{j}-\delta_{i}^{j} \pi_{0}^{0}\right)+\omega^{*} \wedge\left(\omega_{*}^{j}-\pi_{*}^{j}\right) .\right.
$$

Cartan's lemma gives us

$$
\begin{aligned}
\pi_{i}^{j}-\delta_{i}^{j} \pi_{0}^{0} & =\omega_{i}^{j}-\delta_{i}^{j} \omega_{0}^{0}+B_{i k}^{j} \omega^{k}+B_{i}^{j} \omega^{*}, \\
\pi_{*}^{j} & =\omega_{*}^{j}+B_{k}^{j} \omega^{k}+B^{j} \omega^{*}
\end{aligned}
$$

with $B_{i k}^{j}=B_{k i}^{j}$. Multiplying (4.12) by $g_{j k}$, adding $g_{i j}$ - times the conjugate of (4.12) with $i$ replaced by $k$, and using (4.5) and (4.10), we find that $B_{i k}^{j}=0$ and $B_{i \bar{k}}=-B_{\bar{k} i}$. Thus the $\mathrm{g}_{1}$ - and the remaining $\mathrm{g}_{0}$-components of $\pi$ are given by

$$
\begin{gathered}
\pi_{i}^{j}-\delta_{i}^{j} \pi_{0}^{0}=\omega_{i}^{j}-\delta_{i}^{j} \omega_{0}^{0}+B_{i}^{j} \omega^{*}, \\
\pi_{i}^{0}=\omega_{i}^{0}+i B_{i k^{-}} \bar{\omega}^{k}-i B_{i} \omega^{*} .
\end{gathered}
$$

The exterior derivative of (4.10) may be written as

$$
0=\omega^{i} \wedge\left(\omega_{i}^{0}-\pi_{i}^{0}\right)+2 \omega^{*} \wedge\left(\omega_{*}^{0}-\pi_{*}^{0}\right)+\bar{\omega}^{i} \wedge\left(\bar{\omega}_{i}^{0}-\bar{\pi}_{i}^{0}\right) .
$$

Now using Cartan's lemma and (4.15), we find that the $g_{2}$-component of $\pi$ is given by

$$
\pi_{*}^{0}=\omega_{*}^{0}-\frac{1}{2} i B_{i} \omega^{i}+\frac{1}{2} i B_{k} \bar{\omega}^{k}+B \omega^{*},
$$

where $B$ is real.

5. The equations of Gauss and Codazzi. We will now find relations between the curvature forms $\Pi$ and $\Omega$. Taking the exterior derivative of (4.14) yields

$$
\begin{aligned}
\Omega_{i}^{j}+\left\{d B_{i}^{j}\right. & \left.+B_{i}^{j}\left(\omega_{0}^{0}-\omega_{*}^{*}\right)\right\} \wedge \omega^{*} \\
= & \Pi_{i}^{j}+\pi_{i}^{P} \wedge \pi_{P}^{j}+\omega^{j} \wedge\left(\omega_{i}^{0}-\pi_{i}^{0}\right)+\omega_{i}^{*} \wedge\left(\pi_{*}^{j}-\omega_{*}^{j}\right)+\pi_{i}^{k} \wedge \pi_{k}^{j} \\
& \quad-\omega_{i}^{k} \wedge \omega_{k}^{j}-i B_{i}^{j} g_{k t} \omega^{k} \wedge \bar{\omega}^{t}-\delta_{i}^{j}\left\{\omega^{k} \wedge\left(\pi_{k}^{0}-\omega_{k}^{0}\right)+\omega^{*} \wedge\left(\pi_{*}^{0}-\omega_{*}^{0}\right)\right\} .
\end{aligned}
$$

Substituting (4.13) and (4.15) into (5.1) and simplifying the resulting expression, we find that

$$
\begin{aligned}
\Omega_{k}^{j}+D B_{i}^{j} \wedge \omega^{*}= & \Pi_{k}^{j}+\pi_{i}^{P} \wedge \pi_{P}^{j}-i\left(\delta_{l}^{j} B_{i k}+\delta_{i}^{j} B_{l k}+g_{i k} B_{l}^{j}+g_{l k} B_{i}^{j}\right) \omega^{t} \wedge \bar{\omega}^{k} \\
& +i\left(\delta_{l} B_{i}+\frac{1}{2} \delta_{i}^{j} B_{l}\right) \omega^{t} \wedge \omega^{*}+i\left(g_{i l} B^{j}+\frac{1}{2} \delta_{i}^{j} B_{l}\right) \bar{\omega}^{l} \wedge \omega^{*}
\end{aligned}
$$

where the covariant differential of $B_{i}^{j}$ is defined by

$$
D B_{i}^{j}=d B_{i}^{j}-B_{k}^{j} \omega_{i}^{k}+B_{i}^{k} \omega_{k}^{j}+B_{i}^{j}\left(\omega_{0}^{0}-\omega_{*}^{*}\right) .
$$


Setting $D B_{i j}-=g_{t j}-D B_{i}^{t}$, we lower the index $j$ in (5.2) and use (4.11) to obtain

$$
\begin{aligned}
\Omega_{i j}^{-}+D B_{i j} \wedge \omega^{*}= & \Pi_{i j}-\left\{H_{i l}^{P} \bar{H}_{j k}^{P}+i\left(g_{l j}-B_{i \bar{k}}+g_{i j} B_{l \bar{k}}+g_{i k} B_{l j}+g_{l k} B_{i j}^{-}\right)\right\} \omega^{t} \wedge \bar{\omega}^{k} \\
& -\left\{H_{i l}^{P} \bar{H}_{j}^{P}-i\left(g_{t j}-B_{i}+\frac{1}{2} g_{i j}-B_{t}\right)\right\} \omega^{t} \wedge \omega^{*} \\
& +\left\{H_{i}^{P} \bar{H}_{j k}^{P}+i\left(g_{i t} B_{j}^{-}+\frac{1}{2} g_{i j}-B_{l}\right)\right\} \bar{\omega}^{t} \wedge \omega^{*} .
\end{aligned}
$$

We now take the exterior derivative of (4.13) and substitute (4.11) and (4.14) through (4.16) when this is possible. The result is

$$
\begin{aligned}
\Omega_{*}^{j}+ & D B_{k}^{j} \wedge \omega^{k}+D B^{j} \wedge \omega^{*} \\
= & \Pi_{*}^{j}+\frac{1}{2} i \delta_{k}^{j} B_{l} \omega^{k} \wedge \omega^{t}-\left\{g^{j \bar{m}} H_{k}^{P} \bar{H}_{m t}^{P}+i\left(g_{k \bar{t}} B^{j}+\frac{1}{2} \delta_{k}^{j} B_{l}\right)\right\} \omega^{k} \wedge \bar{\omega}^{t} \\
& +g^{j \bar{m}} H^{P} \bar{H}_{m t}^{P} \bar{\omega}^{1} \wedge \omega^{*}+\left(B_{k}^{i} B_{i}^{j}-\delta_{k}^{j} B-g^{j \bar{m}} H_{k}^{P} \bar{H}_{m}^{P}\right) \omega^{k} \wedge \omega^{*}
\end{aligned}
$$

where we have set

$$
D B^{i}=d B^{i}+B^{j} \omega_{j}^{i}-2 B_{k}^{i} \omega_{*}^{k}+B^{i}\left(\omega_{0}^{0}-2 \omega_{*}^{*}\right) .
$$

Similar considerations involving the exterior derivative of (4.16) yield

$$
\begin{aligned}
\Omega_{*}^{0}-\frac{1}{2} i D B_{i} \wedge & \omega^{i}+\frac{1}{2} i D B_{j}^{-} \wedge \bar{\omega}^{j}+D B \\
= & \Pi_{*}^{0}-i\left(H_{k}^{P} \bar{H}_{l}^{P}+i B_{k}^{j} B_{j t}^{-}-g_{k i} B\right) \omega^{k} \wedge \bar{\omega}^{t}-i\left(H_{k}^{P} \bar{H}^{P}+B_{k}^{j} B_{j}\right) \omega^{k} \wedge \omega^{*} \\
& +i\left(\bar{H}_{l}^{P} H^{P}-B^{j} B_{j t}\right) \bar{\omega}^{t} \wedge \omega^{*},
\end{aligned}
$$

where

$$
D B=d B+\frac{3}{2} i B_{k} \omega_{*}^{k}-\frac{3}{2} i B^{k} \omega_{k}^{0}+2 B\left(\omega_{0}^{0}-\omega_{*}^{*}\right) .
$$

We define the covariant derivatives of the functions $B_{i j}, B_{i}, B$, with respect to the connection $\omega$, by means of the following formulae:

$$
\begin{gathered}
D B_{i j}=B_{i j, k} \omega^{k}+B_{i j, t} \bar{\omega}^{t}+B_{i j, *} \omega^{*}, \\
D B_{i}=B_{i, k} \omega^{k}+B_{i, \bar{t}} \bar{\omega}^{t}+B_{i, *} \omega^{*}, \\
D B=B_{, k} \omega^{k}+B_{, i} \bar{\omega}^{t}+B_{, *} \omega^{*} .
\end{gathered}
$$

(5.10) Proposition. Let $S_{A \bar{B} \mu \bar{\nu}}$ be the components of the curvature tensor defined by setting $\Omega_{A \bar{B}}=S_{A \bar{B} \mu \bar{\nu}} \omega^{\mu} \wedge \omega^{\bar{\nu}}$. Then between the functions $S_{A \bar{B} \mu \bar{\nu}}$ and $C_{A \bar{B} \mu \bar{\nu}}$, the coefficients of the second fundamental form, and the coefficients $B_{i j}, B_{i}, B$ and their covariant derivatives we have the following relations:

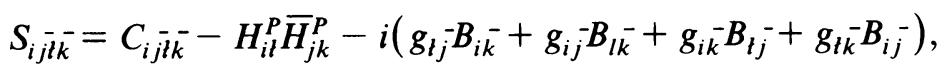

$$
\begin{aligned}
& S_{i j k \bar{*}}=C_{i j \bar{k} \bar{*}}-H_{i k}^{P} \bar{H}_{j}^{P}-B_{i j, k}+i\left(g_{k j}-B_{i}+\frac{1}{2} g_{i j} B_{k}\right) \text {, } \\
& S_{* i \bar{j} \bar{k}}=C_{* \overline{i j \bar{k}}}-H_{j}^{P} \bar{H}_{i}^{P}+B_{j i, *}^{-}-B_{i, j}^{-}+B_{j}^{k} B_{k i}^{-} \text {, }
\end{aligned}
$$




$$
\begin{aligned}
& S_{* i *_{j}}=C_{* i \psi_{j}}-H^{P} \bar{H}_{i j}^{P}+\bar{B}_{i, j}, \\
& S_{* \bar{*} \bar{k}}=C_{* \bar{j} j \bar{*}}-H_{j}^{P} \bar{H}^{P}-B_{j}^{k} B_{k}-\frac{1}{2} B_{j, *}+i B_{, *} .
\end{aligned}
$$

Furthermore $B_{j, k}=B_{k, j}$ and $B_{i j, k}-B_{k j, i}=\frac{1}{2} i\left(g_{k j}-B_{i}-g_{i j} B_{k}\right)$.

Proof. The first two equations can be obtained directly from (5.4); the second two from (5.5). Since $\Omega_{* \bar{*}}=-i \Omega_{*}^{0}$, the fifth equation is a consequence of (5.7). The final two equations may be obtained from the second and fourth using the symmetries of the curvature tensors and those of the coefficients of the second fundamental form.

Applying the trace normalizations on $\Omega$ and $\Pi$ to the first four equations of (5.10), one obtains

(5.11) Proposition. The coefficients $B_{i j}, B_{k}, B$ are completely determined by the coefficients of the second fundamental form. We have:

$$
\begin{aligned}
B_{i j} & =\frac{i}{n+2}\left(H_{i k}^{P} H_{P j}^{k-}-\frac{1}{2(n+1)} g_{i j}-H_{t k}^{P} H_{P}^{i k}\right), \\
B_{i} & =-2 i /(n+2)\left\{B_{j, i}^{j}+H_{k i}^{P} H_{P}^{k}\right\}, \\
B & =1 / n\left(B_{j, *}^{j}-B_{, j}^{j}+B_{i}^{j} B_{j}^{i}-H_{i}^{P} H_{P}^{i}\right) .
\end{aligned}
$$

The classical equation of Gauss in Riemannian geometry gives the relationship between the Riemannian curvature tensor of an isometrically immersed submanifold, the curvature tensor of the ambient space, and the second fundamental form of the immersion. The preceding propositions obviously allow us to write down the pseudoconformal analogues of this equation. One relation of the desired type, which is the simplest in that it involves no covariant derivatives of the $B$ 's, is obtained by substituting the first equation of (5.11) into the first equation of (5.10), viz.

$$
\begin{aligned}
& S_{i \bar{j} \hat{k} \bar{k}}=C_{i j \bar{t} \bar{k}}-H_{i t}^{P} H_{j P \bar{k}} \\
& +(n+2)^{-1}\left(g_{l j} H_{i m}^{P} H_{P k}^{m}+g_{i j} H_{l m}^{P} H_{P k}^{m}+g_{i \bar{k}} H_{l m}^{P} H_{P j}^{m_{j}}+g_{l \bar{k}} H_{i m}^{P} H_{P \bar{j}}^{m}\right) \\
& -(n+1)^{-1}(n+2)^{-1}\left(g_{t j} g_{i k}+g_{i j} g_{t k}\right) H_{m i}^{P} H_{P}^{m i} \text {. }
\end{aligned}
$$

As this is the only one of these equations we will need, we leave to the reader the straightforward computation by which the $B$ 's may be eliminated from the remaining equations of (5.10).

Although the result is true for $n=1$, we only obtain the following from (5.12).

(5.13) THEOREM. Let $V$ be a space with a normal pseudoconformal connection and $M$ a pseudoconformally immersed submanifold of dimension $2 n+1, n>1$. If $V$ is pseudoconformally flat and $M$ is auto-parallel in $V$, then $M$ is also flat.

The classical Codazzi equations of Riemannian geometry relate the covariant derivatives of the second fundamental form of a submanifold with the normal components of the ambient curvature tensor. The pseudoconformal analogues are 
easily derived. To this end define the covariant differentials of the coefficients of the second fundamental form, with respect to the connection $\pi$, as follows:

$$
\begin{aligned}
& D H_{i j}^{P}=d H_{i j}^{P}-H_{k j}^{P} \pi_{i}^{k}+H_{i k}^{Q} \pi_{Q}^{P}-H_{i k}^{P} \pi_{j}^{k}+H_{i j}^{P} \pi_{0}^{0}, \\
& D H_{i}^{P}=d H_{i}^{P}-H_{j}^{P} \pi_{i}^{j}+H_{i}^{Q} \pi_{Q}^{P}-H_{i j}^{P} \pi_{*}^{j}+H^{P}\left(\pi_{0}^{0}-\pi_{*}^{*}\right), \\
& D H^{P}=d H^{P}-2 H_{j}^{P} \pi_{*}^{j}+H^{Q} \pi_{Q}^{P}+H^{P}\left(\pi_{0}^{0}-2 \pi_{*}^{*}\right) .
\end{aligned}
$$

(5.15) Proposition. Between the covariant differentials (5.14) and the normal components of the curvature of $\pi$, we have the relations

$$
\begin{gathered}
D H_{i j}^{P} \wedge \omega^{j}+D H_{i}^{P} \wedge \omega^{*}=-i\left(H_{i}^{P} g_{j k}+g_{i k}-H_{j}^{P}\right) \omega^{j} \wedge \bar{\omega}^{k}+i g_{i j}-H^{P} \bar{\omega}^{j} \wedge \omega^{*}+\Pi_{i}^{P}, \\
D H_{j}^{P} \wedge \omega^{j}+D H^{P} \wedge \omega^{*}=i H^{P} g_{j k} \omega^{j} \wedge \bar{\omega}^{k}+\Pi_{*}^{P} .
\end{gathered}
$$

Proof. Substituting the first equation of (4.11) into the relation obtained by taking its exterior derivative, one sees, using (4.13) and (4.14), that the first two equations of (5.14) are precisely what one needs to make the first equation above hold. The second relation is obtained by similarly considering the exterior derivative of the second equation of (4.11).

The covariant derivatives of the $H$ 's are defined as follows:

$$
\begin{aligned}
& D H_{i j}^{P}=H_{i j, k}^{P} \omega^{k}+H_{i j, i}^{P} \bar{\omega}^{l}+H_{i j, *}^{P} \omega^{*}, \\
& D H_{i}^{P}=H_{i, k}^{P} \omega^{k}+H_{i, i}^{P} \bar{\omega}^{l}+H_{i, *}^{P} \omega^{*}, \\
& D H^{P}=H_{, k}^{P} \omega^{k}+H_{, i}^{P} \bar{\omega}^{l}+H_{, *}^{P} \omega^{*} .
\end{aligned}
$$

From (5.15) we immediately get the equations

$$
\begin{array}{ll}
H_{i j, \bar{k}}^{P}=i\left(H_{i}^{P} g_{j \bar{k}}+g_{i \bar{k}} H P_{j}\right)+C_{i j \bar{k}}^{P-}, & H_{j, \bar{k}}^{P}=i g_{j k} H^{P}-C_{j * \bar{k}}^{P}, \\
H_{i j, k}^{P}=H_{i k, j}^{P}, & H_{i j, *}^{P}=H_{i, j}^{P}+C_{i j *}^{P} .
\end{array}
$$

Now contracting the indices $j, \bar{k}$ in the first two equations of (5.17) and using the trace normalization on $\Pi$ gives us

(5.18) Proposition. The coefficients $H_{i}^{P}$ and $H^{P}$ of the second fundamental form are completely determined by the coefficients $H_{i j}^{P}$ and their covariant derivatives; namely

$$
H_{i}^{P}=-(i /(n+1)) H_{i j}^{P j}, \quad H^{P}=-(i / n) H_{i,}^{P i} .
$$

(5.19) Corollary. $M$ is auto-parallel in $V$ if and only if the coefficients $H_{i j}^{P}$ vanish identically.

6. The induced connection. In the previous section we derived the basic equations relating the intrinsic connections $\omega$ and $\pi$. The purpose of this section is to introduce an extrinsic pseudoconformal connection on $M$ which is induced by $\pi$ and to study its relationship with $\omega$. We define $\phi$ locally to be the matrix of 1-forms on $M$ whose entries are given by

$$
\phi_{J}^{K}=\pi_{J}^{K}+(n+2)^{-1} \delta_{J}^{K} \pi_{P}^{P}
$$


(6.2) Proposition. $\phi$ defines a pseudoconformal connection on $M$, called the induced connection.

Proof. First note that $\phi_{J}^{J}=0$ and $\phi_{J}^{K} g_{K \bar{L}}+g_{J K} \phi_{\bar{L}} \overline{\bar{K}}=0$, by the corresponding relations for $\pi$, so that $\phi$ is $s u(n+1,1)$-valued. Now let $U$ be a change of the adapted $Q$-frame field which leaves the normal frames fixed, i.e. $U$ is given by (4.2) with $t_{P}^{Q}=\delta_{P}^{Q}$. Under $U, \pi$ undergoes the transformation $\tilde{\pi}_{A}^{B} U_{B}^{C}=U_{A}^{B} \pi_{B}^{C}+d U_{A}^{C}$. In particular, $\tilde{\pi}_{P}^{Q}=\pi_{P}^{Q}$. Consequently,

$$
\begin{aligned}
\tilde{\phi}_{J}^{K} U_{K}^{L} & =\left(\tilde{\pi}_{J}^{K}+(n+2)^{-1} \delta_{J}^{K} \tilde{\pi}_{P}^{P}\right) U_{K}^{L}=\tilde{\pi}_{J}^{B} U_{B}^{L}+(n+2)^{-1} \tilde{\pi}_{P}^{P} U_{J}^{L} \\
& =U_{J}^{B} \pi_{B}^{L}+d U_{J}^{L}+(n+2)^{-1} \pi_{P}^{P} U_{J}^{L} \\
& =U_{J}^{B}\left(\pi_{B}^{L}+(n+2)^{-1} \delta_{P}^{P} \pi_{B}^{L}\right)+d U_{J}^{L}=U_{J}^{K} \phi_{K}^{L}+d U_{J}^{L},
\end{aligned}
$$

so that $\phi$ does indeed define a pseudoconformal connection.

REMARK. We should perhaps, at this point, discuss the dependence of the connection $\phi$ on the choice of the normal frame field $Z_{P}$. For this purpose it is convenient to broaden our definition of a pseudoconformal connection.

Note that the indefinite unitary group $\mathcal{G}=U(n+1,1)$ acts transitively on the real hyperquadric $Q$ in $\mathbf{P}^{n+1}$. Let $\mathcal{H}$ denote an isotropy subgroup of $\mathcal{G}$. We now consider Cartan connections of type $\mathcal{G} / \mathcal{H}$, i.e. we allow $u(n+1,1)$-valued connection 1 -forms on principal $\mathcal{H}$-bundles. This being said, we could agree to call a connection as in (2.1) a special pseudoconformal connection.

Because the action of $\mathcal{G}$ on $Q$ is not even almost effective we must introduce a notion of equivalence for these general pseudoconformal connections. Since what really concerns us are the projective transformations between infinitesimally close tangent hyperquadrics, it is natural to call two pseudoconformal connections equivalent if they determine the same linear fractional transformations of $Q$. It is apparent that this will be the case if and only if their difference takes values in the Lie algebra of the center of $\mathcal{G}$.

It is not hard to see that each pseudoconformal connection is equivalent to a unique special pseudoconformal connection. In fact, the connections $\psi=\left(\pi_{J}^{K}\right)$ and $\phi$ defined by (6.1) are equivalent. Now under a change of the normal frames by a transformation $U$, as in (4.2) with $t=1, t_{i}^{j}=\delta_{i}^{j}, t_{i}=\tau=\tau^{j}=0$, we easily find that $\psi$ is invariant. In this way we have avoided the computation which shows directly that the transform $\tilde{\phi}$, of $\phi$ by $U$, is equivalent to $\phi$. In any case, we have now shown that the induced pseudoconformal connection is independent of the choice of normal frame field. (This is to be contrasted with the situation in the geometry of submanifolds in a space with a projective connection. It is the existence of the Levi form which makes the behavior of our geometry closer to that of the conformal case [11].)

The curvature form of $\phi$ is, of course, given by the structure equation $d \phi_{J}^{K}=\phi_{J}^{L} \wedge$ $\phi_{L}^{K}+\Phi_{J}^{K}$; we may write $\Phi_{J}^{K}=R_{J \rho \bar{\sigma}}^{K} \omega^{\rho} \wedge \omega^{\bar{\sigma}}$. A straightforward computation using 
(6.1) and (4.11) yields

(6.3) Proposition. The induced connection $\phi$ is torsionfree, i.e. $\Phi_{0}^{*}=\Phi_{0}^{i}=0$. Moreover:

$$
\begin{aligned}
\Phi_{0}^{0} & =(n+2)^{-1} d \pi_{P}^{P} \\
& =-(n+2)^{-1}\left\{\Pi_{i}^{i}-g^{i \bar{j}}\left(H_{i k}^{P} \omega^{k}+H_{i}^{P} \omega^{*}\right) \wedge\left(H_{j P \bar{t}} \overline{\omega^{t}}+H_{j P}^{-} \omega^{*}\right)\right\}, \\
\Phi_{i}^{j} & =\Pi_{i}^{j}+\delta_{i}^{j} \Pi_{0}^{0}-g^{\overline{j t}}\left(H_{i k}^{P} \omega^{k}+H_{i}^{P} \omega^{*}\right) \wedge\left(H_{t P \bar{m}} \omega^{\bar{m}}+H_{t P} \omega^{*}\right), \\
\Phi_{i}^{0} & =\Pi_{i}^{0}-i\left(H_{i j}^{P} \omega^{j}+H_{i}^{P} \omega^{*}\right) \wedge\left(H_{k P}^{-} \omega^{\bar{k}}+H_{P} \omega^{*}\right), \\
\Phi_{*}^{0} & =\Pi_{*}^{0}-i\left(H_{j}^{P} \omega^{j}+H^{P} \omega^{*}\right) \wedge\left(H_{\bar{k} P}^{-} \omega^{\bar{k}}+H_{P} \omega^{*}\right) .
\end{aligned}
$$

For the components $R_{J \rho \bar{\sigma}}^{K}$ of the curvature tensor we have:

$$
\begin{gathered}
R_{i k \bar{l}}^{j}=C_{i k \bar{t}}^{j-}+\delta_{i}^{j} C_{0 k \bar{l}}^{0}-g^{j \bar{m}} H_{i k}^{P} H_{\bar{m} \overline{P l}}, \\
R_{i j \bar{k}}^{0}=C_{i j \bar{k}}^{0}-H_{i j}^{P} H_{\bar{k} P}^{-}, \quad R_{* j \bar{k}}^{0}=C_{* j \bar{k}}^{0}-i H_{j}^{P} H_{\bar{k} P}^{-} .
\end{gathered}
$$

Computing the traces of each of the curvature forms in (6.3) we immediately arrive at the following conclusion.

(6.5) THEOREM. The induced connection on $M$ coincides with the intrinsic (normal) connection if and only if the following equations hold along $M$ :

$$
\begin{aligned}
d \pi_{P}^{P} & =0, & & i H_{i j}^{P} H_{P}^{j}=C_{i j}^{0 j}, \\
i H_{j}^{P} H_{P}^{j} & =C_{* j}^{0 j}, & H_{i j}^{P} H_{P}^{j k} & =C_{i j}^{k j} .
\end{aligned}
$$

(6.6) THEOREM. If $M$ is a hypersurface in $V$ (i.e. $p=1)$, then the induced connection on $M$ is normal if and only if $M$ is auto-parallel in $V$ and on $M$ we have

$$
C_{i k}^{j k}=C_{i k}^{0 k}=C_{* k}^{0 k}=0 .
$$

Proof. Note that for a hypersurface

$$
d \pi_{n+1}^{n+1}=\pi_{n+1}^{i} \wedge \pi_{i}^{n+1}=-g^{i \bar{k}}\left(\bar{H}_{k j}^{n+1} \bar{\omega}^{j}+\bar{H}_{k}^{n+1} \omega^{*}\right) \wedge\left(H_{i t}^{n+1} \omega^{t}+H_{i}^{n+1} \omega^{*}\right) .
$$

Now if the induced connection is normal the first equation of (6.5) implies, in particular, that $g^{i \bar{k}} H_{i j}^{n+1} \bar{H}_{k t}^{n+1}=0$. Since $g^{i \bar{k}}$ is nondegenerate, $H_{i j}^{n+1}=0$. By (5.19) $M$ is auto-parallel in $V$. The conditions on $C_{A \mu \bar{\nu}}^{B}$ now follow from (5.18) and the last three equations of (6.5). The converse is evident.

Theorem (6.6) implies the following unpublished result of S. Kobayashi.

(6.7) COROLlARY. The induced pseudoconformal connection on a hypersurface $M$ in a pseudoconformally flat space $V$ is normal if and only if $M$ is auto-parallel in $V$.

Our final result is a consequence of (6.5), the first equation of (6.3), and (5.12).

(6.8) THEOREM. The induced connection on an auto-parallel pseudoconformally immersed submanifold of a pseudoconformally flat space is normal, hence flat. 
The author wishes to express his appreciation for the encouragement and many helpful suggestions offered him by his thesis advisor, Professor S. Kobayashi.

The results presented here formed part of a doctoral dissertation submitted to the University of California, Berkeley in 1979.

\section{BIBLIOGRAPHY}

1. D. Burns, Jr. and S. Shnider, Real hypersurfaces in complex manifolds, Proc. Sympos. Pure Math., vol. 30, Amer. Math. Soc., Providence, R. I., 1977, pp. 141-168.

2. E. Cartan, Sur la géométrie pseudo-conforme des hypersurfaces de deux variables complexes. I, Ann. Mat. Pura Appl. 11 (1932), 17-90 (or Oeuvres II, 2, 1231-1304); II, Ann. Scuola Norm. Sup. Pisa Cl. Sci. 1 (1932), 333-354 (or Oeuvres III, 2, 1217-1238).

3. S.-S. Chern and J. K. Moser, Real hypersurfaces in complex manifolds, Acta Math. 133 (1974), 219-271.

4. S. Helgason, Differential geometry and symmetric spaces, Academic Press, New York, 1962.

5. S. Kobayashi, Transformation groups in differential geometry, Springer-Verlag, Berlin and New York, 1972.

6. S. Kobayashi and K. Nomizu, Foundations of differential geometry, Vols. I, II, Wiley, New York, $1963,1969$.

7. N. Tanaka, On non-degenerate real hypersurfaces, graded Lie algebras and Cartan connections, Japan J. Math. 2 (1976), 131-190.

8. S. M. Webster, Pseudo-hermitian structures on a real hypersurface, Trans. Amer. Math. Soc. 231 (1977), 179-190.

9.

10. K. Yamaguchi, Non-degenerate real hypersurfaces in complex manifolds admitting large groups of pseudo-conformal transformations. I, Nagoya Math. J. 62 (1976), 55-96.

11. K. Yano and Y. Muto, Sur la théorie des espaces à connexion conforme normale et la géométrie conforme des espaces de Riemann, J. Fac. Sci. Tokyo Imp. Univ. 4 (1941), 117-169.

Department of Mathematics, University of Chicago, Chicago, Illinois 60637

Current address: Department of Mathematics, Statistics and Computer Science, University of Illinois at Chicago, Chicago, Illinois 60680 